\title{
A SUPER-EARTH ORBITING THE NEARBY SUN-LIKE STAR HD 1461
}

\author{
Eugenio J. Rivera ${ }^{1}$, R. Paul Butler ${ }^{2}$, Steven S. Vogt ${ }^{1}$, Gregory Laughlin ${ }^{1}$, Gregory W. Henry ${ }^{3}$, \\ and Stefano Meschiari ${ }^{1}$ \\ ${ }^{1}$ UCO/Lick Observatory, University of California, Santa Cruz, CA 95064, USA \\ 2 Department of Terrestrial Magnetism, Carnegie Institution of Washington, 5241 Broad Branch Road, NW, Washington, DC 20015-1305, USA \\ ${ }^{3}$ Center of Excellence in Information Systems, Tennessee State University, Nashville, TN 37209, USA \\ Received 2009 September 23; accepted 2009 November 23; published 2009 December 22
}

\begin{abstract}
We present precision radial velocity (RV) data that reveal a Super-Earth mass planet and two probable additional planets orbiting the bright nearby G0V star HD 1461. Our 12.8 years of Keck High Resolution Echelle Spectrometer precision RVs indicate the presence of a $7.4 M_{\oplus}$ planet on a 5.77 day orbit. The data also suggest, but cannot yet confirm, the presence of outer planets on low-eccentricity orbits with periods of 446.1 and 5017 days, and projected masses $(M \sin i)$ of 27.9 and $87.1 M_{\oplus}$, respectively. Test integrations of systems consistent with the $\mathrm{RV}$ data suggest that the configuration is dynamically stable. We present a 12.2 year time series of photometric observations of HD 1461, which comprise 799 individual measurements, and indicate that it has excellent longterm photometric stability. However, there are small amplitude variations with periods comparable to those of the suspected second and third signals in the RVs near 5000 and 446 days, thus casting some suspicion on those periodicities as Keplerian signals. If the 5.77 day companion has a Neptune-like composition, then its expected transit depth is of order $d \sim 0.5 \mathrm{mmag}$. The geometric a priori probability of transits is $\sim 8 \%$. Phase folding of the ground-based photometry shows no indication that transits of the 5.77 day companion are occurring, but highprecision follow-up of HD 1461 during upcoming transit phase windows will be required to definitively rule out or confirm transits. This new system joins a growing list of solar-type stars in the immediate galactic neighborhood that are accompanied by at least one Neptune (or lower) mass planets having orbital periods of 50 days or less.
\end{abstract}

Key words: planetary systems - stars: individual (HD 1461)

\section{INTRODUCTION}

Over 400 extrasolar planets are now known. The majority of these have been discovered by using precision radial velocities (RVs) to detect the reflex barycentric motion of the host star. We have had a large sample of over 1000 nearby stars under precision RV survey for the past 13 years at Keck with the High Resolution Echelle Spectrometer (HIRES). One of the target stars is HD 1461, a nearby G0V star, only $23.4 \pm 0.5 \mathrm{pc}$ away (Perryman et al. 1997). This star has been on the Keck program since 1996 October. Over the past 12.8 years, we have accumulated a total of 164 precision RVs that indicate a system of at least two planets orbiting this star. In this paper, we present all of these RV data and discuss the planetary system that they imply.

\section{BASIC PROPERTIES OF THE HOST STAR HD 1461}

HD 1461 (HIP 1499, HR 72, GJ 16.1, GJ 9009, BD -0838, SAO 128690, SPOCS 13$)$ is a bright $(V=6.46)$ and wellstudied star. It has been characterized in a number of studies, including those of Valenti \& Fischer (2005), Takeda et al. (2007), and Sousa et al. (2008). Table 1 summarizes recent determinations of the fundamental stellar parameters for HD 1461. Taken together, these properties indicate that it is an old, metal-rich, inactive star well suited for precision RV planet searches. Using $\mathrm{Ca} \mathrm{H}+\mathrm{K}$ measurements taken between 1994 and 2006, Hall et al. (2007) found that HD 1461 is one of the 13 targets for which the observed variability is zero within the uncertainties. In 51 observations over eight seasons, they measured a mean $\log R_{\mathrm{HK}}^{\prime}$ of -5.04 . Hall et al. (2009) found a mean value of -5.00 over seven seasons with seasonal averages ranging from -4.96 to -5.01 . Wright et al. (2004) found a mean value of -5.03 , along with an estimated slow rotational period of 29 days and an age of $6.3 \mathrm{Gyr}$. Our measurement of $\log R_{\mathrm{HK}}^{\prime}=$ -5.00 leads to an estimate (Wright 2005) of $1.59 \mathrm{~m} \mathrm{~s}^{-1}$ for the expected RV jitter due to stellar surface activity. The age of HD 1461 was estimated as $4.2_{-2.1}^{+1.7}$ Gyr by Valenti \& Fischer (2005) and 7.12 $2_{-1.56}^{+1.40} \mathrm{Gyr}$ by Takeda et al. (2007). The large discrepancy and uncertainties for these chromospheric ages is not surprising since the correlation between age and chromospheric activity becomes very weak for ages bigger than 2 Gyr (see, e.g., Pace \& Pasquini 2004). In summary, HD 1461 is a nearby, bright star with physical properties that are quite similar to our own Sun, and is an ideal candidate star for the application of highprecision Doppler velocity monitoring.

\section{OBSERVATIONS}

The HIRES (Vogt et al. 1994) of the Keck I telescope was used to monitor HD 1461. A total of 164 Keck observations were obtained, from 1996 October 10 to 2009 August 10, a data span of 4687 days. The median internal velocity uncertainty for these Keck data is $0.72 \mathrm{~m} \mathrm{~s}^{-1}$.

Doppler shifts were measured in the usual manner (Butler et al. 1996) by placing an iodine absorption cell just ahead of the spectrometer slit in the converging beam from the telescope. This gaseous iodine absorption cell superimposes a rich forest of iodine lines on the stellar spectrum, providing a wavelength calibration and proxy for the point-spread function (PSF) of the spectrometer. The iodine cell is sealed and temperaturecontrolled to $50 \pm 0.1{ }^{\circ} \mathrm{C}$ such that the column density of iodine remains constant. For the Keck planet search program, we operate the HIRES at a spectral resolving power of $R \approx 70,000$ and a wavelength range of $3700-8000 \AA$, though only the region $5000-6200 \AA$ (with iodine lines) is used in the present Doppler 
Table 1

Stellar Parameters for HD 1461

\begin{tabular}{|c|c|c|}
\hline Parameter & Value & Reference \\
\hline Spec. type & G0V & Cenarro et al. (2007) \\
\hline \multirow[t]{3}{*}{$\operatorname{Mass}\left(M_{\odot}\right)$} & $1.08 \pm 0.04$ & Valenti \& Fischer (2005) \\
\hline & $1.026_{-0.030}^{+0.040}$ & Takeda et al. (2007) \\
\hline & $1.018 \pm 0.1$ & Sousa et al. (2008) \\
\hline \multirow[t]{2}{*}{ Radius $\left(R_{\odot}\right)$} & $1.095 \pm 0.026$ & Valenti \& Fischer (2005) \\
\hline & $1.11 \pm 0.04$ & Takeda et al. (2007) \\
\hline \multirow[t]{2}{*}{ Luminosity $\left(L_{\odot}\right)$} & $1.197 \pm 0.113$ & Valenti \& Fischer (2005) \\
\hline & $1.188 \pm 0.017$ & Sousa et al. (2008) \\
\hline Distance $(\mathrm{pc})$ & $23.4 \pm 0.5$ & Perryman et al. (1997) \\
\hline$V \sin i\left(\mathrm{~km} \mathrm{~s}^{-1}\right)$ & 1.6 & Valenti \& Fischer (2005) \\
\hline \multirow{4}{*}{$\log R_{\mathrm{HK}}^{\prime}$} & -5.03 & Wright et al. (2004) \\
\hline & -5.04 & Hall et al. (2007) \\
\hline & -5.00 & Hall et al. (2009) \\
\hline & -5.00 & This work \\
\hline$P_{\text {rot }}$ (days) & 29 & Wright et al. (2004) \\
\hline \multirow[t]{3}{*}{ Age (Gyr) } & 6.3 & Wright et al. (2004) \\
\hline & $4.2_{-2.1}^{+1.7}$ & Valenti \& Fischer (2005) \\
\hline & $7.12_{-1.56}^{+1.40}$ & Takeda et al. (2007) \\
\hline \multirow[t]{3}{*}[\mathrm{Fe}/\mathrm{H}]{} & 0.18 & Valenti \& Fischer (2005) \\
\hline & $0.20 \pm 0.01$ & Cenarro et al. (2007) \\
\hline & $0.19 \pm 0.01$ & Sousa et al. (2008) \\
\hline \multirow[t]{2}{*}{$T_{\text {eff }}(\mathrm{K})$} & $5765 \pm 18$ & Valenti \& Fischer (2005); Sousa et al. (2008) \\
\hline & 5808 & Cenarro et al. (2007) \\
\hline \multirow[t]{3}{*}{$\log g$} & $4.37 \pm 0.03$ & Valenti \& Fischer (2005); Takeda et al. (2007) \\
\hline & 4.39 & Cenarro et al. (2007) \\
\hline & $4.38 \pm 0.03$ & Sousa et al. (2008) \\
\hline
\end{tabular}

analysis. The iodine region is divided into $\sim 700$ chunks of $2 \AA$ each. Each chunk produces an independent measure of the wavelength, PSF, and Doppler shift. The final measured velocity is the weighted mean of the velocities of the individual chunks.

Table 2 lists the complete set of 164 RVs for HD 1461, corrected for the solar system barycenter. The table lists the barycentric JD of observation center, RV, and internal uncertainty. The internal uncertainty reflects only one term in the overall error budget, and results from a host of systematic errors from characterizing and determining the PSF, detector imperfections, optical aberrations, effects of under-sampling the iodine lines, etc. Two additional major sources of error are photon statistics and stellar jitter. The latter varies widely from star to star, and can be mitigated to some degree by selecting magnetically inactive older stars and by time-averaging over the star's unresolved low-degree surface $p$-modes. For most of the past 12.8 years, only single exposures at Keck were taken of HD 1461 at each epoch. Since these single exposures were much shorter than the characteristic timescale of low-degree surface $p$-modes on the star, they suffered from additional noise (stellar jitter). By 2008 July at Keck, we began $p$-mode averaging each observation, combining multiple shots of HD 1461 over a 510 minute dwell at each epoch. All observations have been further binned on two-hour timescales.

Although all of the observations used in this work were obtained with the HIRES at the Keck I telescope, 28 of the RVs in Table 2 were derived from publicly available spectra from the NASA-Keck Observatory Archive. These velocities are marked with "Q01" in the observatory column in Table 2. The CCD format of the Q01 run was shifted by several angstroms relative to our long-term standard, introducing a zero-point velocity offset between Q01 and our standard setup. This is an additional parameter to be determined in the fits discussed below.
Table 2

Radial Velocities for HD 1461

\begin{tabular}{|c|c|c|c|}
\hline $\begin{array}{c}\text { Barycentric JD } \\
(-2450000) \\
\end{array}$ & $\begin{array}{c}\mathrm{RV} \\
\left(\mathrm{m} \mathrm{s}^{-1}\right)\end{array}$ & $\begin{array}{c}\text { Error } \\
\left(\mathrm{m} \mathrm{s}^{-1}\right)\end{array}$ & Observatory \\
\hline 366.93641 & -0.21 & 1.36 & $\mathrm{~K}$ \\
\hline 715.00792 & -9.17 & 1.11 & $\mathrm{~K}$ \\
\hline 785.76804 & -14.14 & 1.22 & $\mathrm{~K}$ \\
\hline 1010.09403 & -5.98 & 1.17 & $\mathrm{~K}$ \\
\hline 1068.92515 & -5.81 & 1.14 & $\mathrm{~K}$ \\
\hline 1173.72568 & -0.48 & 1.10 & $\mathrm{~K}$ \\
\hline 1343.08899 & -2.04 & 1.12 & $\mathrm{~K}$ \\
\hline 1374.10891 & -4.86 & 1.27 & $\mathrm{~K}$ \\
\hline 1438.88093 & -3.75 & 1.55 & $\mathrm{~K}$ \\
\hline 1550.73862 & -4.91 & 1.25 & $\mathrm{~K}$ \\
\hline 1883.80712 & -0.32 & 1.34 & $\mathrm{~K}$ \\
\hline 2098.12817 & -7.96 & 1.51 & $\mathrm{~K}$ \\
\hline 2236.72882 & -0.08 & 1.30 & $\mathrm{~K}$ \\
\hline 2489.05617 & -2.67 & 1.51 & $\mathrm{~K}$ \\
\hline 2537.92834 & -3.48 & 1.57 & $\mathrm{~K}$ \\
\hline 2575.84461 & -1.98 & 1.54 & $\mathrm{~K}$ \\
\hline 2829.05914 & -7.47 & 1.43 & $\mathrm{~K}$ \\
\hline 2899.03253 & -2.51 & 1.37 & $\mathrm{~K}$ \\
\hline 3196.06321 & 2.86 & 1.28 & $\mathrm{~K}$ \\
\hline 3238.01691 & 0.50 & 0.21 & $\mathrm{~K}$ \\
\hline 3238.90057 & 2.88 & 0.42 & $\mathrm{~K}$ \\
\hline 3239.94328 & 6.80 & 0.44 & $\mathrm{~K}$ \\
\hline 3240.99951 & 6.49 & 0.49 & $\mathrm{~K}$ \\
\hline 3241.11480 & 7.06 & 0.65 & $\mathrm{~K}$ \\
\hline 3301.85378 & -4.94 & 0.65 & $\mathrm{~K}$ \\
\hline 3302.81034 & -0.65 & 0.61 & $\mathrm{~K}$ \\
\hline 3338.75610 & 1.71 & 1.08 & $\mathrm{~K}$ \\
\hline 3339.75947 & -1.18 & 0.66 & $\mathrm{~K}$ \\
\hline 3369.75405 & -6.98 & 0.64 & $\mathrm{~K}$ \\
\hline 3397.70379 & 3.24 & 0.58 & $\mathrm{~K}$ \\
\hline 3398.73620 & -0.30 & 0.61 & $\mathrm{~K}$ \\
\hline 3399.69869 & -2.37 & 0.64 & $\mathrm{~K}$ \\
\hline 3400.70946 & 4.84 & 0.64 & $\mathrm{~K}$ \\
\hline 3547.12033 & 3.03 & 0.63 & $\mathrm{~K}$ \\
\hline 3548.10688 & 3.59 & 0.96 & $\mathrm{~K}$ \\
\hline 3549.13414 & 0.06 & 1.14 & $\mathrm{~K}$ \\
\hline 3550.11494 & 3.57 & 0.61 & $\mathrm{~K}$ \\
\hline 3551.12904 & 4.00 & 0.62 & $\mathrm{~K}$ \\
\hline 3552.12582 & 4.68 & 0.58 & $\mathrm{~K}$ \\
\hline 3603.04546 & 7.42 & 0.63 & $\mathrm{~K}$ \\
\hline 3604.06836 & 10.32 & 0.75 & $\mathrm{~K}$ \\
\hline 3605.04697 & 6.44 & 0.64 & $\mathrm{~K}$ \\
\hline 3692.92330 & -2.41 & 0.61 & $\mathrm{~K}$ \\
\hline 3693.84499 & 1.79 & 0.54 & $\mathrm{~K}$ \\
\hline 3694.78313 & 1.37 & 0.58 & $\mathrm{~K}$ \\
\hline 3695.70050 & 4.03 & 0.53 & $\mathrm{~K}$ \\
\hline 3695.82845 & 4.85 & 0.59 & $\mathrm{~K}$ \\
\hline 3696.76371 & 5.63 & 0.57 & $\mathrm{~K}$ \\
\hline 3723.82034 & -1.81 & 0.60 & $\mathrm{~K}$ \\
\hline 3724.74409 & 1.77 & 0.63 & $\mathrm{~K}$ \\
\hline 3746.73490 & -1.19 & 0.69 & $\mathrm{~K}$ \\
\hline 3747.79465 & -0.46 & 0.97 & $\mathrm{~K}$ \\
\hline 3748.72482 & 1.91 & 0.68 & $\mathrm{~K}$ \\
\hline 3749.72744 & -0.78 & 0.62 & $\mathrm{~K}$ \\
\hline 3775.71584 & -2.90 & 0.67 & $\mathrm{~K}$ \\
\hline 3776.70867 & 2.10 & 0.65 & $\mathrm{~K}$ \\
\hline 3777.71251 & 1.53 & 0.80 & $\mathrm{~K}$ \\
\hline 3778.71106 & -2.19 & 0.86 & $\mathrm{~K}$ \\
\hline 3779.73772 & -2.38 & 0.81 & $\mathrm{~K}$ \\
\hline 3927.10455 & 0.42 & 0.62 & $\mathrm{~K}$ \\
\hline 3928.05300 & 1.31 & 0.85 & $\mathrm{~K}$ \\
\hline 3959.10907 & -1.33 & 0.62 & $\mathrm{~K}$ \\
\hline 3961.04337 & 0.02 & 0.64 & $\mathrm{~K}$ \\
\hline 3962.01510 & 2.99 & 0.61 & $\mathrm{~K}$ \\
\hline 3981.90046 & -3.83 & 0.65 & $\mathrm{~K}$ \\
\hline
\end{tabular}


Table 2

(Continued)

\begin{tabular}{|c|c|c|c|}
\hline $\begin{array}{c}\text { Barycentric JD } \\
(-2450000)\end{array}$ & $\begin{array}{c}\mathrm{RV} \\
\left(\mathrm{m} \mathrm{s}^{-1}\right)\end{array}$ & $\begin{array}{c}\text { Error } \\
\left(\mathrm{m} \mathrm{s}^{-1}\right)\end{array}$ & Observatory \\
\hline 3982.98884 & 2.04 & 0.66 & $\mathrm{~K}$ \\
\hline 3983.87130 & 2.26 & 0.58 & $\mathrm{~K}$ \\
\hline 3984.97007 & 6.59 & 0.65 & $\mathrm{~K}$ \\
\hline 4083.81187 & 2.61 & 0.73 & $\mathrm{~K}$ \\
\hline 4084.77778 & 3.39 & 0.73 & $\mathrm{~K}$ \\
\hline 4129.74967 & 8.16 & 0.66 & $\mathrm{~K}$ \\
\hline 4131.71246 & 4.76 & 0.75 & $\mathrm{~K}$ \\
\hline 4279.11113 & 0.46 & 1.19 & $\mathrm{~K}$ \\
\hline 4280.10709 & -1.69 & 1.26 & $\mathrm{~K}$ \\
\hline 4286.10589 & -5.34 & 1.22 & $\mathrm{~K}$ \\
\hline 4295.08454 & -2.01 & 1.15 & $\mathrm{~K}$ \\
\hline 4305.06828 & -5.52 & 0.61 & $\mathrm{~K}$ \\
\hline 4306.02305 & -1.97 & 0.83 & $\mathrm{~K}$ \\
\hline 4306.99662 & -3.53 & 0.94 & $\mathrm{~K}$ \\
\hline 4307.10265 & -0.77 & 0.83 & $\mathrm{~K}$ \\
\hline 4308.07916 & -0.27 & 0.71 & $\mathrm{~K}$ \\
\hline 4309.07893 & 0.58 & 0.85 & $\mathrm{~K}$ \\
\hline 4310.02797 & -4.99 & 0.80 & $\mathrm{~K}$ \\
\hline 4310.12123 & -2.37 & 1.19 & $\mathrm{~K}$ \\
\hline 4311.00946 & -6.55 & 0.84 & $\mathrm{~K}$ \\
\hline 4311.10912 & -2.30 & 1.23 & $\mathrm{~K}$ \\
\hline 4312.00404 & -4.29 & 0.82 & $\mathrm{~K}$ \\
\hline 4312.11147 & -2.84 & 0.82 & $\mathrm{~K}$ \\
\hline 4313.00139 & -3.43 & 0.87 & $\mathrm{~K}$ \\
\hline 4313.10575 & -2.31 & 0.86 & $\mathrm{~K}$ \\
\hline 4313.99949 & -0.07 & 0.85 & $\mathrm{~K}$ \\
\hline 4314.10977 & 0.60 & 0.90 & $\mathrm{~K}$ \\
\hline 4315.12175 & -1.56 & 0.86 & $\mathrm{~K}$ \\
\hline 4319.01064 & 3.70 & 0.70 & $\mathrm{~K}$ \\
\hline 4336.05526 & 1.63 & 0.75 & $\mathrm{~K}$ \\
\hline 4337.10146 & 4.33 & 0.81 & $\mathrm{~K}$ \\
\hline 4343.93370 & 5.91 & 1.21 & $\mathrm{~K}$ \\
\hline 4396.76696 & -2.48 & 1.18 & $\mathrm{~K}$ \\
\hline 4397.82001 & -4.10 & 1.23 & $\mathrm{~K}$ \\
\hline 4398.84808 & -2.28 & 1.12 & $\mathrm{~K}$ \\
\hline 4427.86323 & 0.36 & 0.67 & $\mathrm{~K}$ \\
\hline 4429.77428 & 2.62 & 1.30 & $\mathrm{~K}$ \\
\hline 4430.77076 & 2.35 & 1.24 & $\mathrm{~K}$ \\
\hline 4454.82508 & -2.21 & 1.20 & $\mathrm{~K}$ \\
\hline 4456.80064 & -2.24 & 1.25 & $\mathrm{~K}$ \\
\hline 4460.78595 & -4.89 & 1.35 & $\mathrm{~K}$ \\
\hline 4461.79834 & -6.06 & 1.13 & $\mathrm{~K}$ \\
\hline 4464.80653 & 4.89 & 1.37 & $\mathrm{~K}$ \\
\hline 4492.70875 & 5.97 & 1.29 & $\mathrm{~K}$ \\
\hline 4634.11567 & -6.90 & 0.92 & $\mathrm{~K}$ \\
\hline 4635.07070 & -5.12 & 0.88 & $\mathrm{~K}$ \\
\hline 4636.09364 & -3.98 & 0.87 & $\mathrm{~K}$ \\
\hline 4637.10870 & -6.08 & 0.88 & $\mathrm{~K}$ \\
\hline 4638.11047 & -2.74 & 0.91 & $\mathrm{~K}$ \\
\hline 4639.09420 & -4.49 & 0.88 & $\mathrm{~K}$ \\
\hline 4640.10790 & -4.80 & 0.84 & $\mathrm{~K}$ \\
\hline 4641.10062 & -7.37 & 0.86 & $\mathrm{~K}$ \\
\hline 4642.07466 & 1.06 & 0.94 & $\mathrm{~K}$ \\
\hline 4644.12501 & 1.89 & 0.89 & $\mathrm{~K}$ \\
\hline 4717.94972 & -2.96 & 0.83 & $\mathrm{~K}$ \\
\hline 4719.00539 & 0.38 & 0.73 & $\mathrm{~K}$ \\
\hline 4720.00787 & -4.48 & 0.66 & $\mathrm{~K}$ \\
\hline 4720.96693 & -4.91 & 0.77 & $\mathrm{~K}$ \\
\hline 4721.98581 & -5.73 & 0.79 & $\mathrm{~K}$ \\
\hline 4722.89844 & -0.54 & 0.71 & $\mathrm{~K}$ \\
\hline 4723.96804 & 0.62 & 0.73 & $\mathrm{~K}$ \\
\hline 4724.96948 & -0.12 & 0.82 & $\mathrm{~K}$ \\
\hline 5022.12042 & -5.09 & 0.52 & $\mathrm{~K}$ \\
\hline 5023.08514 & -3.59 & 0.85 & $\mathrm{~K}$ \\
\hline 5024.11008 & -1.22 & 0.49 & $\mathrm{~K}$ \\
\hline
\end{tabular}

Table 2

(Continued)

\begin{tabular}{|c|c|c|c|}
\hline $\begin{array}{c}\text { Barycentric JD } \\
(-2450000)\end{array}$ & $\begin{array}{c}\mathrm{RV} \\
\left(\mathrm{m} \mathrm{s}^{-1}\right)\end{array}$ & $\begin{array}{c}\text { Error } \\
\left(\mathrm{m} \mathrm{s}^{-1}\right)\end{array}$ & Observatory \\
\hline 5025.12159 & -4.13 & 0.58 & $\mathrm{~K}$ \\
\hline 5050.06798 & -2.46 & 0.49 & $\mathrm{~K}$ \\
\hline 5051.09955 & -2.43 & 0.46 & $\mathrm{~K}$ \\
\hline 5052.04858 & 1.40 & 0.55 & $\mathrm{~K}$ \\
\hline 5053.08104 & -0.45 & 0.56 & $\mathrm{~K}$ \\
\hline 5054.04263 & 4.05 & 0.59 & $\mathrm{~K}$ \\
\hline 3713.70121 & 0.45 & 0.70 & Q01 \\
\hline 3713.76642 & 1.42 & 0.59 & Q01 \\
\hline 3713.82984 & 3.44 & 0.60 & Q01 \\
\hline 3714.69699 & 0.24 & 0.61 & Q01 \\
\hline 3714.75572 & 0.56 & 0.58 & Q01 \\
\hline 3714.82460 & 0.22 & 0.56 & Q01 \\
\hline 3715.68459 & -1.65 & 0.66 & Q01 \\
\hline 3715.72791 & 0.10 & 0.70 & Q01 \\
\hline 3715.77573 & -2.24 & 0.64 & Q01 \\
\hline 3715.82808 & -0.68 & 0.63 & Q01 \\
\hline 3716.72884 & -3.10 & 0.59 & Q01 \\
\hline 3716.77010 & -1.49 & 0.63 & Q01 \\
\hline 3716.82182 & -1.79 & 0.72 & Q01 \\
\hline 3717.79314 & -4.50 & 0.56 & Q01 \\
\hline 3726.72138 & -1.00 & 0.61 & Q01 \\
\hline 3726.77494 & 2.24 & 0.59 & Q01 \\
\hline 3727.71647 & -1.39 & 0.54 & Q01 \\
\hline 3727.77202 & -4.18 & 0.56 & Q01 \\
\hline 3727.81049 & -2.38 & 0.58 & Q01 \\
\hline 3728.73194 & -5.62 & 0.54 & Q01 \\
\hline 3728.79946 & -2.87 & 0.59 & Q01 \\
\hline 3728.84615 & -2.39 & 0.58 & Q01 \\
\hline 3728.84971 & 0.18 & 0.60 & Q01 \\
\hline 3729.70727 & 1.63 & 0.85 & Q01 \\
\hline 3729.81947 & 0.00 & 0.60 & Q01 \\
\hline 3730.71913 & 2.23 & 0.60 & Q01 \\
\hline 3730.77245 & 2.82 & 0.56 & Q01 \\
\hline 3730.80874 & 2.49 & 0.54 & Q01 \\
\hline
\end{tabular}

\section{PHOTOMETRY}

In addition to our RV observations, we acquired highprecision photometric observations of HD 1461 during 13 consecutive observing seasons from 1996 November to 2009 January with the T8 $0.80 \mathrm{~m}$ automatic photometric telescope (APT) at the Fairborn Observatory. Our APTs can detect shortterm, low-amplitude brightness variability in solar-type stars due to rotational modulation of the visibility of surface magnetic activity (spots and plages), as well as longer-term variations associated with the growth and decay of individual active regions and the occurrence of stellar magnetic cycles (Henry 1999). The photometric observations help us to establish whether observed RV variations are caused by stellar activity or planetary reflex motion (e.g., Henry et al. 2000a). Queloz et al. (2001) and Paulson et al. (2004) have presented several examples of periodic $\mathrm{RV}$ variations in solar-type stars caused by photospheric spots and plages. The photometric observations are also useful to search for transits of the planetary companions (e.g., Henry et al. 2000b; Charbonneau et al. 2000).

The T8 APT is equipped with a two-channel precision photometer that separates the Strömgren $b$ and $y$ passbands with a dichroic filter and takes simultaneous $30 \mathrm{~s}$ integrations with two EMI 9124QB bi-alkali photomultiplier tubes. The APT measures the difference in brightness between a program star and a nearby constant comparison star or stars. The 

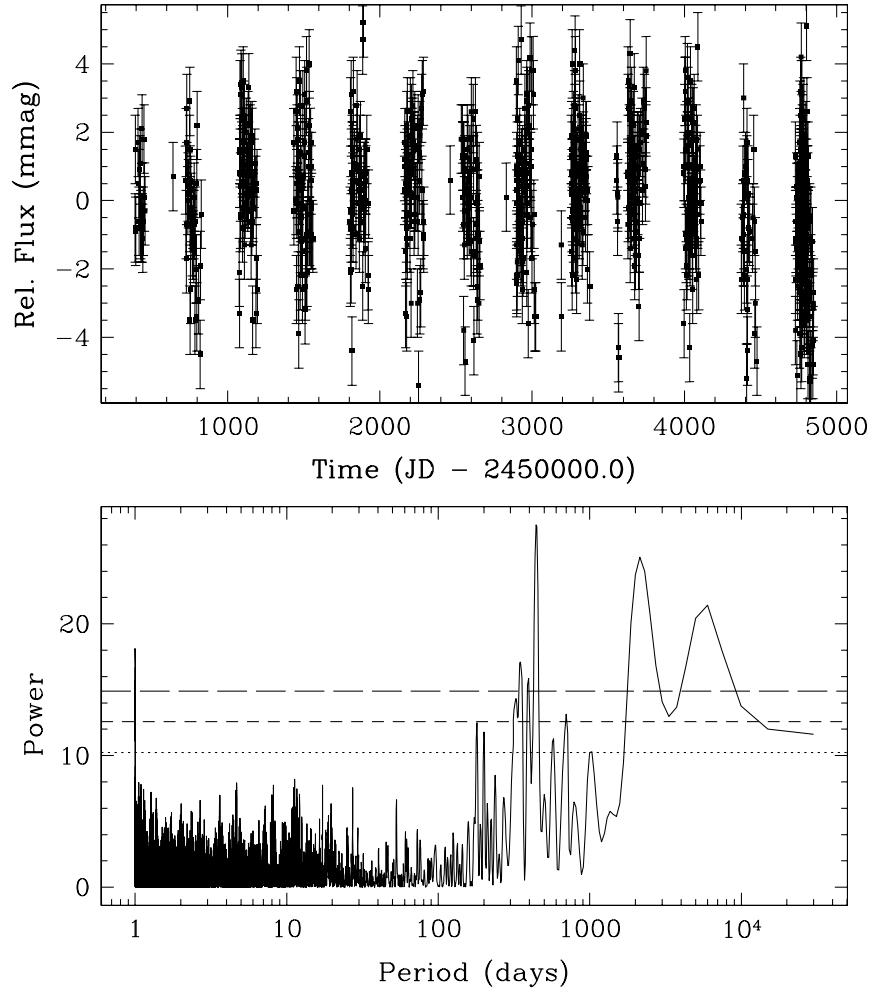

Figure 1. Top: differential photometry of HD 1461. Times are barycentric JD. Bottom: Lomb-Scargle periodogram of the photometry.

typical precision of a single observation is approximately $0.0015 \mathrm{mag}$, as measured for pairs of constant stars. The automatic telescopes, photometers, observing procedures, and data reduction techniques are described in Henry (1999). Further details on the development and operation of the automated telescopes can be found in Henry (1995a, 1995b) and Eaton et al. (2003).

For HD 1461, we used HD $2361(V=7.89, B-V=0.470$, F2) as our primary comparison star. The individual Strömgren $b$ and $y$ differential magnitudes have been corrected for differential extinction with nightly extinction coefficients and transformed to the Strömgren system with yearly mean transformation coefficients. Since HD 1461 lies at a declination of $-8^{\circ}$, the photometric observations from Fairborn were made at airmass 1.3-1.8, which is somewhat higher than most observations. Therefore, to improve the precision of the measurements, we combined the Strömgren $b$ and $y$ differential magnitudes into a single $(b+y) / 2$ passband.

A total of 799 differential magnitudes from 13 observing seasons are plotted in the top panel of Figure 1. The data scatter about their mean with a standard deviation of $\sigma=0.0019$ mag, which provides an upper limit to possible brightness variation in HD 1461. The data are plotted such that observations that are brighter/dimmer than the mean are indicated with positive/negative flux values. A Lomb-Scargle periodogram of the photometric measurements is shown in the bottom panel of Figure 1 and reveals a significant periodicity within the data at 444.5 days with an estimated false alarm probability (FAP) of $3.3 \times 10^{-9}$ (calculated using procedures described in Gilliland \& Baliunas (1987) and Cumming (2004)). This peak is near the tallest peak in the RV periodogram of the residuals of the circular two-planet fit discussed below. A second peak spans from 3000 to 9000 days with an estimated FAP of

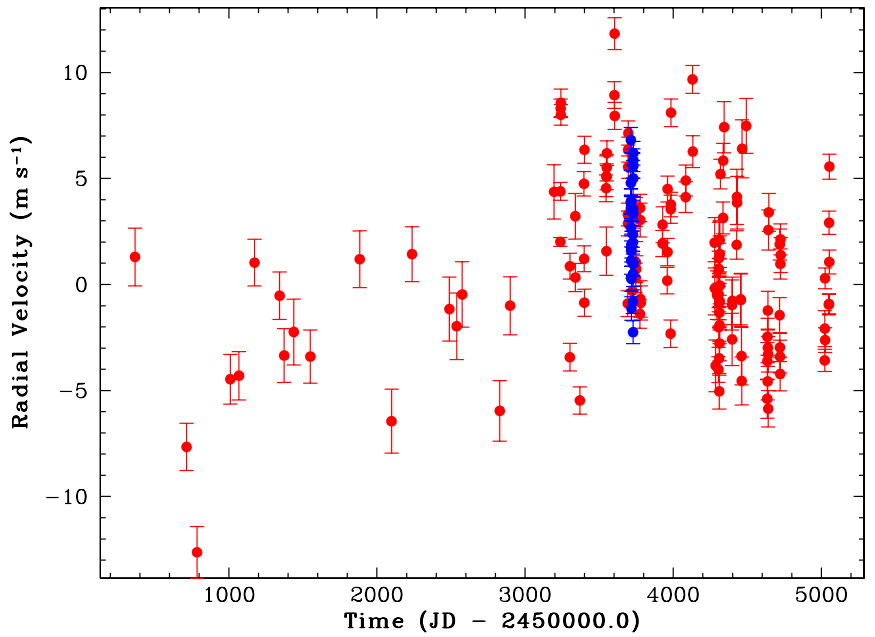

Figure 2. Relative RVs of HD 1461. Velocities based on spectra obtained by our group are in red, and those based on archived Keck spectra (Q01) are in blue.

$2.1 \times 10^{-6}$. This broad peak is similar to the one present in the periodogram of the residuals of the (circular) one-planet fit also discussed below. The three horizontal lines in the bottom panel of Figure 1 and in all similar periodograms below represent FAPs of $0.1 \%, 1 \%$, and $10 \%$ from top to bottom, respectively. We computed least-squares sine fits for the three RV planet candidates described below. Sinusoid fits with periods 5.77, 446.1, and 5017 days yield semi-amplitudes of only 0.00018 , 0.00070 , and $0.00061 \mathrm{mag}$, respectively. We conclude that the lack of significant coherent photometric variability of HD 1461 supports planetary reflex motion as the cause of the 5.77 day periodicity in the RV measurements. The weak periodicities observed at 445 and 3000-9000 days, on the other hand, do warrant some caution in interpreting the RV periodicities at those periods as being due to planetary companions.

\section{THE PLANETARY SYSTEM ORBITING HD 1461}

The RVs show a rms scatter of $3.80 \mathrm{~m} \mathrm{~s}^{-1}$ about the mean velocity. This significantly exceeds the combined scatter due to the underlying precision of both our measurement pipeline and the scatter expected in this star due to its predicted $1.59 \mathrm{~m} \mathrm{~s}^{-1}$ level of stellar jitter. Figure 2 shows the RV data set from Table 2. An offset of $-1.864 \mathrm{~m} \mathrm{~s}^{-1}$ (Q01 - Keck) has been applied between the two sets. Note that in general, observations prior to 2004 August have larger internal uncertainties. The improvement in the internal uncertainties is a result of the CCD upgrade discussed in some detail in Rivera et al. (2005). Also, the observations prior to 2004 August appear to be offset from those taken after the CCD upgrade. This is an artifact of our sampling with a low-frequency period that is not too distinct from one year during the first few years of our observations. As a result, during this time, we always sampled the period of HD $1461 \mathrm{c}$ such that the star's RV is low.

Figure 3 shows the periodogram of the RV data set (top panel). Power at each sampled period is proportional to the relative improvement (drop in $\chi_{v}^{2}$ ) in the fit quality for a circular model versus a constant velocity model. The periodogram shows a number of significant signals, with the strongest peak occurring at a period of 5.77 days. The FAP of this peak is estimated (adopting the procedure described in Cumming 2004) to be $1.1 \times 10^{-10}$. Furthermore, this 5.77 day signal lies well away from the periods favored by the sampling window (Figure 3, 


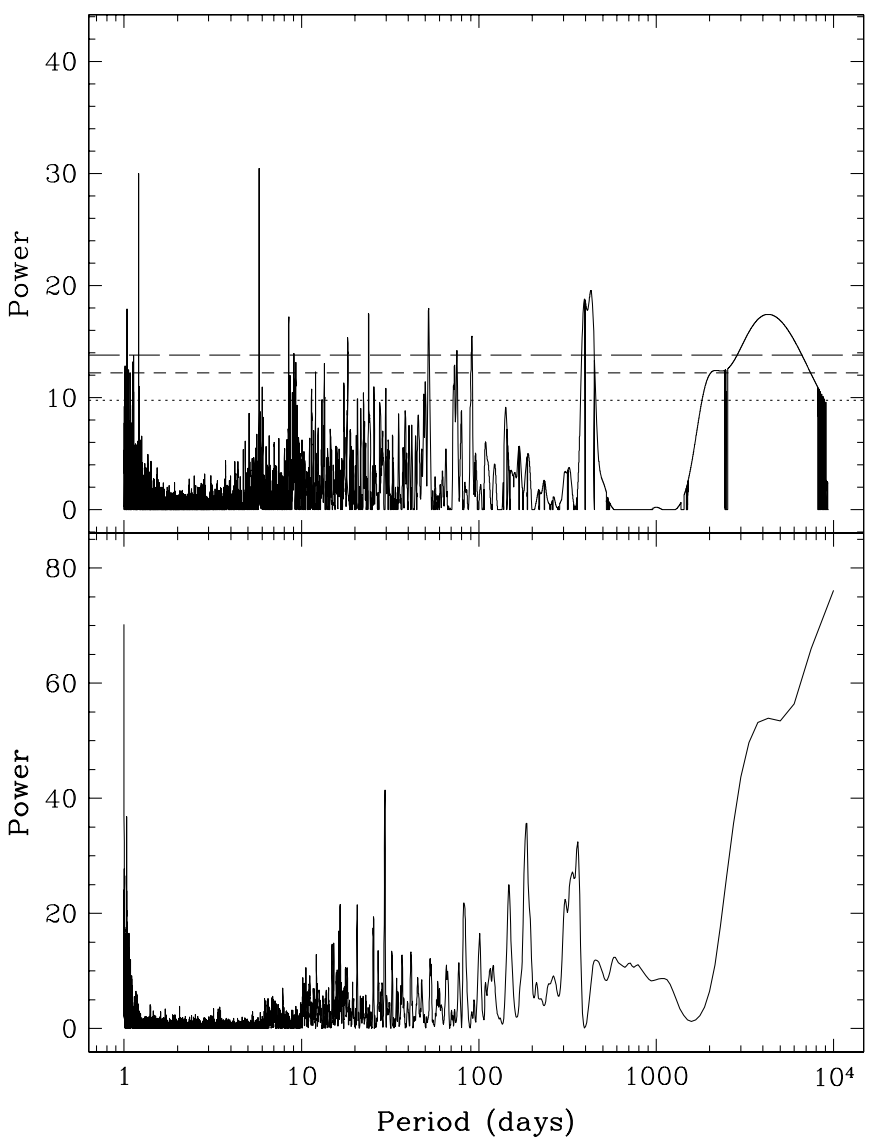

Figure 3. Top: periodogram of the RV data set for HD 1461. The tallest peak is at 5.77 days. Bottom: power spectral window of the RV data.

bottom panel), which produces spurious power at periods near $29.6,186.3,361.5,147.8$, and 82.4 days. Signals near any of these periods would be suspected of being artifacts of the observing scheduling.

Based on the periodogram, with an assumed stellar mass of $1.022 M_{\odot}$, we fit a planet of mass $7.4 M_{\oplus}$ and period 5.77 days on a circular orbit to the RV data. The presence of this planet (with a RV semi-amplitude of $K=2.60 \mathrm{~m} \mathrm{~s}^{-1}$ ) reduces the rms scatter of the velocity residuals to $3.43 \mathrm{~m} \mathrm{~s}^{-1}$. Figure 4 (top panel) shows the one-planet residuals periodogram which has a strong peak at 4226 days. The 4226 day signal has a FAP of $9.9 \times 10^{-16}$ and can be modeled with a companion with $K=2.77 \mathrm{~m} \mathrm{~s}^{-1}$ and a mass of $0.22 M_{\text {Jup }}$. The addition of this planet further reduces the rms scatter to $2.87 \mathrm{~m} \mathrm{~s}^{-1}$.

Figure 4 (bottom panel) shows the two-planet residuals periodogram, assuming circular orbits, which has a peak at a period of 446.1 days. The FAP of the 446.1 day signal is $4.5 \times 10^{-6}$. If Keplerian, this periodicity can be ascribed to the presence of a 446.1 day, $27.9 M_{\oplus}$ companion. This third component has $K=2.30 \mathrm{~m} \mathrm{~s}^{-1}$. The three-planet model, assuming circular orbits, has an rms scatter of $2.41 \mathrm{~m} \mathrm{~s}^{-1}$.

Figure 5 shows the RV model for the three-planet fit with circular orbits with the observations overplotted. It is centered around the time of the high cadence Q01 observations.

Given the one-, two-, and three-planet models, we can look either for solutions in which the planetary orbits are circular or solutions where the eccentricities are allowed to float. Inclusion of eccentricities provides only a modest improvement to the orbital fits, leading us to conclude that a significant amount of additional Doppler velocity monitoring will be required to

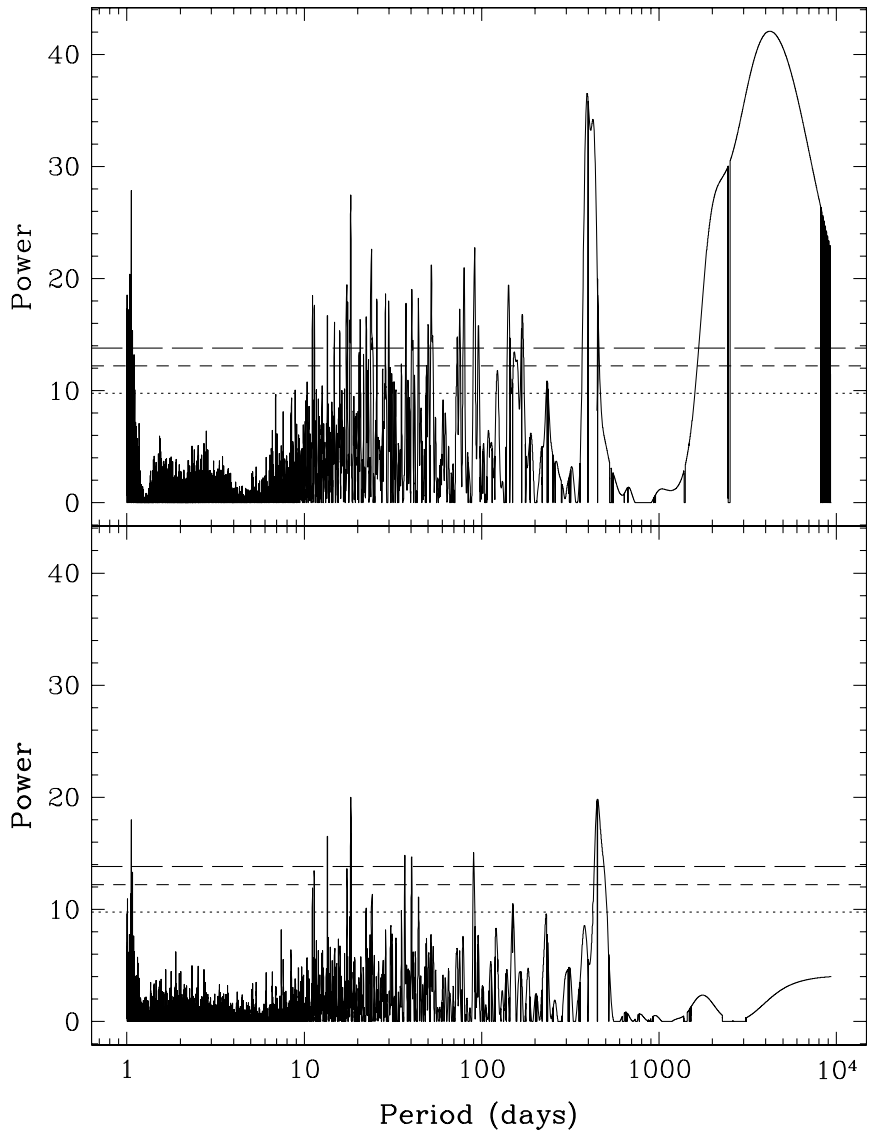

Figure 4. Top: periodogram of the one-planet residuals, assuming a circular orbit, of the RV data set for HD 1461. Bottom: periodogram of the two-planet residuals, assuming circular orbits.

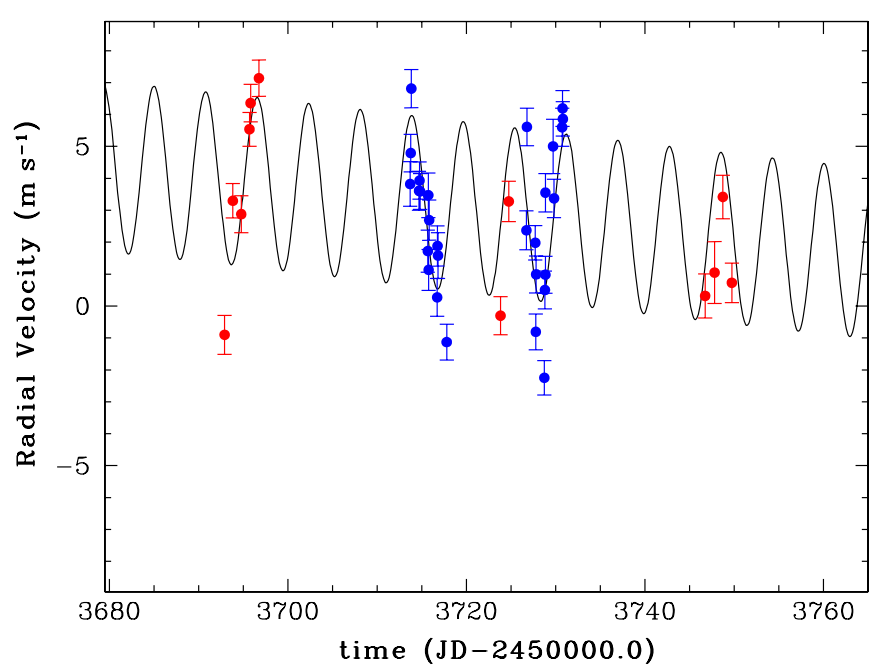

Figure 5. Zoomed-in view of the circular three-planet model for HD 1461 (black curve). Overplotted are the Keck observations in red and the Q01 observations in blue.

improve the eccentricity uncertainties. However, allowing the eccentricities to float for the two-planet fit dramatically reduces the significance of the 446 day periodicity observed in the two-planet residuals (see below). Additionally, if we fit for a second planet with a period in the range $\sim 390-450$ days on an eccentric orbit, the significance of the long-period planet can also be reduced. In Tables 3 and 4, we present our bestfit versions of the system under the assumption of circular 


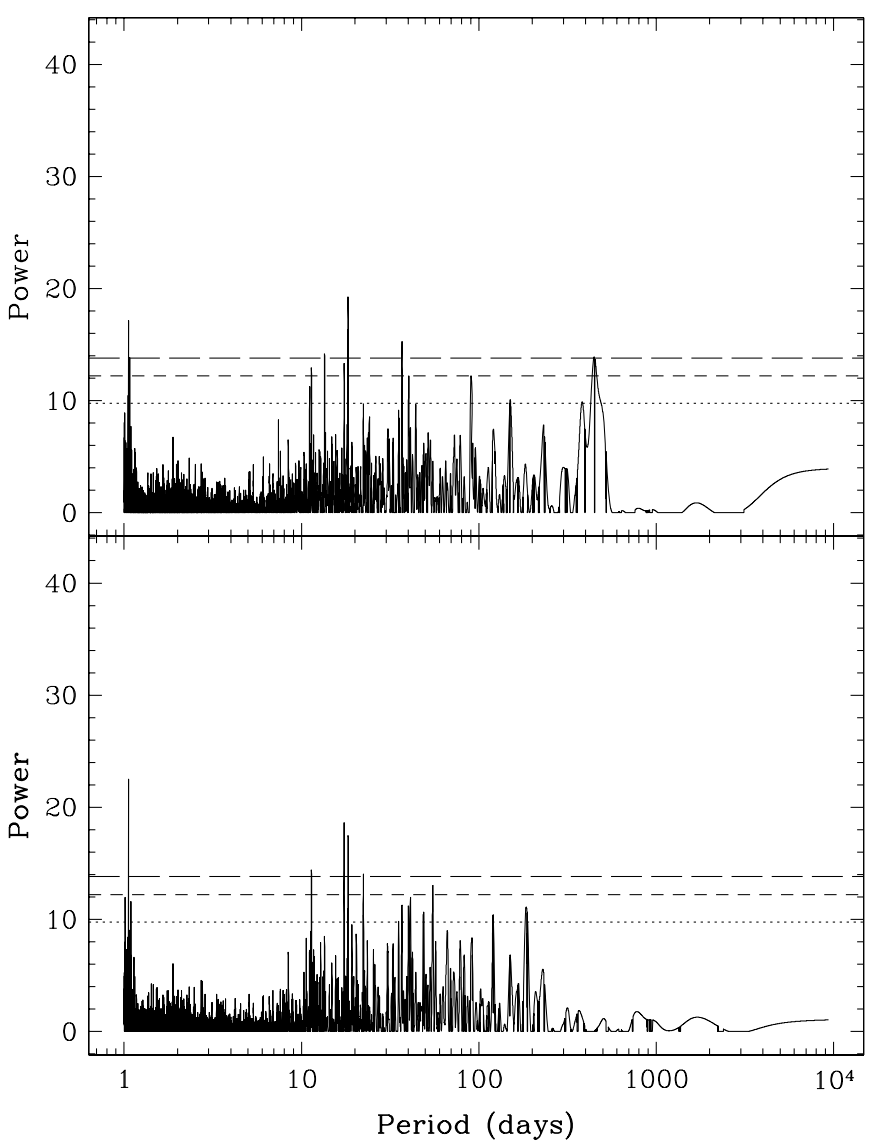

Figure 6. Top: periodogram of the two-planet residuals, with floating eccentricities, of the RV data set for HD 1461. Bottom: periodogram of the three-planet residuals, assuming circular orbits.

orbits (Table 3) and with the additional degrees of freedom provided by fully Keplerian trajectories (Table 4). For the orbital fits, we assume $i=90^{\circ}$ and $\Omega=0^{\circ}$. The inclusion of planet-planet gravitational interactions in the fits were found to be unnecessary. Uncertainties are based on 1000 bootstrap trials following the procedure in Section 15.6 from Press et al. (1992). The standard deviations of the fitted parameters to the bootstrapped RV's were adopted as the uncertainties. The fitted mean anomalies are reported at epoch JD 2450366.936. The mass of the host star is assumed to be $1.022 M_{\odot}$, the mean of the isochrone masses of Sousa et al. $\left(2008 ; 1.018 \pm 0.1 M_{\odot}\right)$ and Takeda et al. $\left(2007 ; 1.026_{-0.030}^{+0.040} M_{\odot}\right)$. Our fitting was carried out with the publicly available Systemic Console (Meschiari et al. 2009).

Figure 6 (top panel) shows the power spectrum of the velocity residuals for the two-planet fit with floating eccentricities. It is clear that the significance of the 446.1 day planet is dramatically reduced.

Figure 6 (bottom panel) shows the power spectrum of the velocity residuals for the three-planet, circular fit. There is a peak near 18.3 days with a FAP of $4.7 \times 10^{-5}$. Although using this period in a four-planet circular fit results in a significant improvement in $\chi_{v}^{2}$, the rms decrease from $2.41 \mathrm{~m} \mathrm{~s}^{-1}$ for the three-planet model to $2.28 \mathrm{~m} \mathrm{~s}^{-1}$ is not significant. Additionally, the fitted amplitude of $1.49 \mathrm{~m} \mathrm{~s}^{-1}$ is significantly smaller than the scatter around the model.

In summary, the Keck HIRES RV data show strong evidence for at least two planets in orbit about the G0V star HD 1461. One of these has a short period of 5.77 days. The other has a large period of about 4000-6000 days, with a

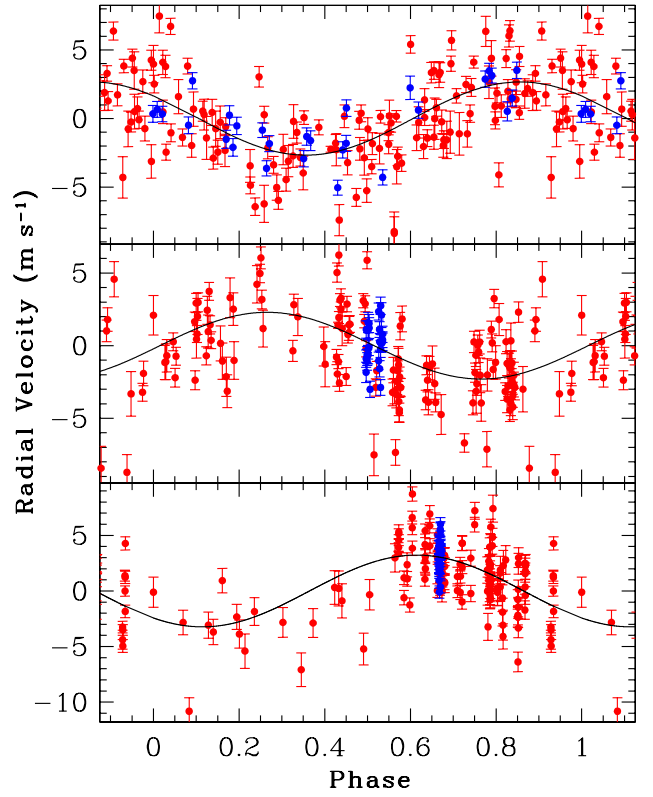

Figure 7. Top: radial velocity of HD 1461 due to planet b folded at 5.77 days. Center: radial velocity of HD 1461 due to planet c folded at 446.1 days. Bottom: radial velocity of HD 1461 due to planet d folded at 5017 days. In each panel, the effect of the other two planets has been subtracted out. The curves represent the model velocities due to each respective planet. The Keck observations are shown in red, and the Q01 velocities are shown in blue.

large uncertainty in both its period and eccentricity. There are also hints of a third planet in the system near 446 days, but the large uncertainties in the parameters of the second planet cast some doubt on the presence of a third planet in the system. Also, if we choose to fit for an eccentric planet with a period of $\sim 390-450$ days, we also find large uncertainties in its parameters. This would cast some doubt on the presence of the large period planet. The close match between the photometric and the Doppler periodicities near 445 days leads us to tread carefully in ascribing a planetary origin to our 446 day RV signal. We note, however, that a 446 day period is too long to be associated with the stellar rotation, given the star's measurable rotational velocity component, $V \sin i=1.6 \mathrm{~km} \mathrm{~s}^{-1}$. If the 446 day Doppler signal does arise from stellar activity, then the periodicity would need to be primarily associated with the lifetimes of the active regions rather than with modulation induced by stellar rotation. Further observations will be needed to refine the parameters of the second planet before a definitive detection claim can be made for a third planet. That said, in Figure 7 we show the barycentric reflex velocity of the host star due to each of three individual companions on circular orbits in the system. In each panel, the velocities are folded at the period of each corresponding planet.

\section{DYNAMICAL ANALYSIS}

It is useful to verify, via numerical integration, whether the planetary configurations listed in Tables 3 and 4 are dynamically stable. Such an analysis is particularly useful in giving rough bounds on the allowed coplanar inclinations relative to the line of sight to Earth. For simulations that do not include the effects of tidal dissipation, Newtonian parameters were used for the initial states for long-term integrations. The MERCURY integration package (Chambers 1999) was used for the simulations with a time step of 0.1 days. The first-order post-Newtonian term in the star's potential was also included, as in Lissauer \& Rivera (2001). 
Table 3

Circular Solutions (Epoch JD 2450366.936)

\begin{tabular}{|c|c|c|c|c|c|c|c|}
\hline Planet & $\begin{array}{l}\text { Period } \\
\text { (d) }\end{array}$ & $\begin{array}{c}K \\
\left(\mathrm{~m} \mathrm{~s}^{-1}\right)\end{array}$ & $e$ & $\begin{array}{c}\omega \\
(\mathrm{deg})\end{array}$ & $\begin{array}{c}M \\
(\mathrm{deg})\end{array}$ & $\begin{array}{c}M \sin i \\
\left(M_{\oplus}\right)\end{array}$ & $\begin{array}{c}a \\
(\mathrm{AU})\end{array}$ \\
\hline \multicolumn{8}{|c|}{ one planet; $\chi_{v}^{2}=19.249 ; \mathrm{rms}=3.43 \mathrm{~m} \mathrm{~s}^{-1}$} \\
\hline HD1461 b & $5.7726 \pm 0.0026$ & $2.6 \pm 0.4$ & 0.0 & $\mathrm{n} / \mathrm{a}$ & $79 \pm 60$ & $7.4 \pm 1.2$ & $0.063437 \pm 0.000019$ \\
\hline \multicolumn{8}{|c|}{ two planets; $\chi_{v}^{2}=13.151 ; \mathrm{rms}=2.87 \mathrm{~m} \mathrm{~s}^{-1}$} \\
\hline HD1461 b & $5.7718 \pm 0.0010$ & $2.8 \pm 0.3$ & 0.0 & $\mathrm{n} / \mathrm{a}$ & $44 \pm 36$ & $7.8 \pm 0.8$ & $0.063431 \pm 0.000008$ \\
\hline HD1461 d & $4019 \pm 1433$ & $2.8 \pm 1.9$ & 0.0 & $\mathrm{n} / \mathrm{a}$ & $285 \pm 47$ & $69.9 \pm 78.9$ & $4.98 \pm 0.99$ \\
\hline \multicolumn{8}{|c|}{ three planets; $\chi_{v}^{2}=10.093 ; \mathrm{rms}=2.41 \mathrm{~m} \mathrm{~s}^{-1}$} \\
\hline HD1461 b & $5.7718 \pm 0.0010$ & $2.7 \pm 0.2$ & 0.0 & $\mathrm{n} / \mathrm{a}$ & $45 \pm 37$ & $7.6 \pm 0.7$ & $0.063431 \pm 0.000008$ \\
\hline HD1461 c & $446 \pm 9$ & $2.3 \pm 0.4$ & 0.0 & $\mathrm{n} / \mathrm{a}$ & $82 \pm 52$ & $27.9 \pm 4.9$ & $1.151 \pm 0.016$ \\
\hline HD1461 d & $5017 \pm 1171$ & $3.2 \pm 1.4$ & 0.0 & $\mathrm{n} / \mathrm{a}$ & $319 \pm 36$ & $87.1 \pm 51.8$ & $5.78 \pm 0.82$ \\
\hline
\end{tabular}

Table 4

Eccentric Solutions (Epoch JD 2450366.936)

\begin{tabular}{|c|c|c|c|c|c|c|c|}
\hline Planet & $\begin{array}{l}\text { Period } \\
\text { (d) }\end{array}$ & $\begin{array}{c}K \\
\left(\mathrm{~m} \mathrm{~s}^{-1}\right)\end{array}$ & $e$ & $\begin{array}{c}\omega \\
(\mathrm{deg})\end{array}$ & $\begin{array}{c}M \\
(\mathrm{deg}) \\
\end{array}$ & $\begin{array}{c}M \sin i \\
\left(M_{\oplus}\right)\end{array}$ & $\begin{array}{c}a \\
(\mathrm{AU})\end{array}$ \\
\hline \multicolumn{8}{|c|}{ one planet; $\chi_{v}^{2}=19.349 ; \mathrm{rms}=3.43 \mathrm{~m} \mathrm{~s}^{-1}$} \\
\hline HD1461 b & $5.7727 \pm 0.0025$ & $2.7 \pm 0.6$ & $0.14 \pm 0.19$ & $58 \pm 69$ & $26 \pm 71$ & $7.6 \pm 1.2$ & $0.063438 \pm 0.000018$ \\
\hline \multicolumn{8}{|c|}{ two planets; $\chi_{v}^{2}=12.768 ; \mathrm{rms}=2.64 \mathrm{~m} \mathrm{~s}^{-1}$} \\
\hline HD1461 b & $5.7720 \pm 0.0011$ & $2.8 \pm 0.4$ & $0.06 \pm 0.14$ & $28 \pm 70$ & $24 \pm 67$ & $8.1 \pm 0.9$ & $0.063432 \pm 0.000008$ \\
\hline HD1461 d & $7000 \pm 200000$ & $4 \pm 24$ & $0.51 \pm 0.24$ & $319 \pm 32$ & $210 \pm 83$ & $101 \pm 357$ & $7 \pm 42$ \\
\hline \multicolumn{8}{|c|}{ three planets; $\chi_{v}^{2}=8.983 ; \mathrm{rms}=2.24 \mathrm{~m} \mathrm{~s}^{-1}$} \\
\hline HD1461 b & $5.7722 \pm 0.0011$ & $2.8 \pm 0.3$ & $0.04 \pm 0.01$ & $186 \pm 63$ & $234 \pm 77$ & $8.1 \pm 0.7$ & $0.063434 \pm 0.000008$ \\
\hline HD1461 c & $454 \pm 4$ & $2.8 \pm 2.7$ & $0.74 \pm 0.13$ & $87 \pm 28$ & $237 \pm 28$ & $22.9 \pm 9.8$ & $1.165 \pm 0.008$ \\
\hline HD1461 d & $5000 \pm 90000$ & $4 \pm 70$ & $0.16 \pm 0.29$ & $326 \pm 79$ & $136 \pm 85$ & $97 \pm 1161$ & $5 \pm 18$ \\
\hline
\end{tabular}

If the three orbits are assumed to be initially circular, with periods, masses and mean anomalies given in Table 3, the system is stable for at least $20 \mathrm{Myr}$. Additionally, assuming the system to be coplanar, if we set the inclination to the sky plane to various values from $i=90^{\circ}$ all the way down to $i=1^{\circ}$ and perform a Newtonian fit for the other nine parameters (three parameters per planet plus the two offsets), $\chi_{v}^{2}$ does not change significantly from the nominal $i=90^{\circ}$ fit. We also find the $i=1^{\circ}$ fit to result in a system that is stable for at least $20 \mathrm{Myr}$. For this inclination, the fitted masses exceed 1.4, 5.1, and $16 M_{\text {Jup }}$. This system is stable because of the small eccentricities. Thus, under the assumption that the system is coplanar and the orbits are (nearly) circular, we cannot place a lower bound on the inclination of the system.

The parameters of the floating-eccentricity version of the HD 1461 system given in Table 4 were also used as the initial input conditions for a $10^{7} \mathrm{yr}$ simulation. The three-planet configuration is disrupted in less than 1.4 Myr.

If additional RV measurements point to secure non-zero eccentricities for the HD 1461 planets, then studies of the long-term dynamical evolution of the system should take the possibility of tidal dissipation in planet $b$ into account.

\section{CHECK FOR TRANSITS BY COMPANION B}

We performed a simple search for transits of planet $\mathrm{b}$ in the HD 1461 photometry. The RVs determine the period of the inner planet to be 5.77 days. The corresponding mean anomaly of the planet, likewise, is $M=79.045$. The stellar radius is taken to be $1.1 R_{\odot}$, the mean of the values in Table 1 .

If HD 1461 b's orbital plane allows for transits, then $\sin i_{b} \sim 1$, and hence $M_{b}=7.4 M_{\oplus}$. If we assume that the planet migrated inward from beyond the ice line, its composition is likely dominated by water. The models of Fortney et al.
(2007) suggest an $R=2.7 R_{\oplus}$ radius for such a planet, leading to a central transit depth of $d \sim 0.05 \%$, or $d \sim 0.5 \mathrm{mmag}$. If the planet has a massive atmosphere, its transit depth will be larger still.

At the time of the first photometric data point, $T_{p 1}=$ JD2450393.7339, our orbital model indicates a mean anomaly $M=310.255$ for planet b. For a circular orbit, transits occur at $M=90^{\circ}$. This means that relative to phase $\phi=0.0$ referenced to $T_{p 1}$, transits are centered at $\phi=0.388$. The planet's orbital velocity is $v_{b} \sim 120 \mathrm{~km} \mathrm{~s}^{-1}$ which, for our $R=1.1 R_{\odot}$ star, implies a transit duration of $\tau=12000 \mathrm{~s}=3.4$ hours. Assuming central transits, planet $\mathrm{b}$ would be observable in transit $2.4 \%$ of the time.

We assumed a simple "top-hat" step-function model for the transit, with the depth, period, and phase given above. The photometric data set contains 799 individual measurements, with $\sigma=0.00188 \mathrm{mag}$. Phase coverage at the 5.77 day period of planet $\mathrm{b}$ is excellent. One thus expects $N=19 \pm 4$ points to lie in the transit window, indicating that, given the data set and the presence of a transiting planet $\mathrm{b}$, one can expect to detect a transit with signal-to-noise ratio, $\mathrm{S} / \mathrm{N} \sim 1$. We therefore conclude that the present photometric data set is insufficient to make a definitive call as to whether transits are occurring. Follow-up with high-precision, high-cadence photometry from either ground or space is therefore warranted.

\section{DISCUSSION}

We present evidence for at least two (and possibly three) low-mass planets orbiting the nearby star HD 1461. With a RV semi-amplitude $K=2.8 \pm 0.3 \mathrm{~m} \mathrm{~s}^{-1}$, the $M \sin i=$ $7.8 \pm 0.8 M_{\oplus}$ inner planet HD $1461 \mathrm{~b}$ is among the very lowestamplitude companions yet detected using the Doppler velocity technique. 
The HD 1461 system is thus another nearby case that joins the emerging population of planets postulated by Mayor et al. (2009), who inferred that about $30 \%$ of solar-type stars in the immediate galactic neighborhood are accompanied by Neptune (or lower) mass planets having orbital periods of 50 days or less.

With a period of only 5.77 days, HD 1461 b has a nonnegligible $P \sim 8 \%$ probability of transiting its parent star. While our phase-folded ground-based photometry does not have the requisite cadence and $\mathrm{S} / \mathrm{N}$ to detect such transits, it would be readily possible to determine whether transits occur by making high-cadence, high-precision observations that span the transit window (see, e.g., Johnson et al. 2009). HD 1461 b's mass is very similar to that of CoRoT-7b, which has recently been determined to have a density similar to that expected for a rocky planet (Queloz et al. 2009). It would be of great interest to learn whether HD $1461 \mathrm{~b}$ is similarly dense, or whether its composition is more reminiscent of ice-giant planets such as Neptune, Gliese 436b, and HAT-P-11b. Such a transit could be detected from space using, for example, the Warm Spitzer platform.

As RV databases grow in the monitoring of chromospherically quiet, nearby stars, systems like HD 1461 are becoming increasingly common. With each new system, the evidence is growing stronger that Super-Earths and other low-mass planets are common around nearby Sun-like and cooler stars. It is thus only a matter of time and adequate cadence before SuperEarth planets are found in the habitable zones of nearby stars. This is only a first reconnaissance of this fascinating and quite nearby system. As RV databases grow for this star, the orbital ephemerides of these planets will become better determined, and more planets will probably be revealed.

We acknowledge the major contributions of fellow members of our previous California-Carnegie Exoplanet team Geoff Marcy, Debra Fischer, Jason Wright, Katie Peek, and Andrew Howard in helping us to obtain the RVs presented in this paper. We thank the anonymous referee who helped improve this work. S.S.V. gratefully acknowledges support from NSF grant AST-0307493, and from the NASA KECK PI program. R.P.B. gratefully acknowledges support from NASA OSS Grant NNX07AR40G, the NASA Keck PI program, the Carnegie Institution of Washington, and the NAI, NASA Astrobiology Institute. G.L. acknowledges support from NSF AST-0449986. G.W.H. acknowledges support from NASA, NSF, Tennessee State University, and the state of Tennessee through its Centers of Excellence program. The work herein is based on observations obtained at the W. M. Keck Observatory, which is operated jointly by the University of California and the California Institute of Technology, and we thank the UC-Keck and NASA-Keck Time Assignment Committees for their support. This research has also made use of the Keck Observatory Archive (KOA), which is operated by the W. M. Keck Observatory and the
NASA Exoplanet Science Institute (NExScI), under contract with the National Aeronautics and Space Administration. We gratefully acknowledge William Cochran for a 10 night data set that has been obtained through KOA and incorporated into this paper. We also wish to extend our special thanks to those of Hawaiian ancestry on whose sacred mountain of Mauna Kea we are privileged to be guests. Without their generous hospitality, the Keck observations presented herein would not have been possible. This research has made use of the SIMBAD database, operated at CDS, Strasbourg, France.

Facilities: Keck (HIRES)

\section{REFERENCES}

Butler, R. P., Marcy, G. W., Williams, E., McCarthy, C., Dosanjh, P., \& Vogt, S. S. 1996, PASP, 108, 500

Chambers, J. E. 1999, MNRAS, 304, 793

Charbonneau, D., Brown, T. M., Latham, D. W., \& Mayor, M. 2000, ApJ, 529, L45

Cenarro, A. J., et al. 2007, MNRAS, 374, 664

Cumming, A. 2004, MNRAS, 354, 1165

Eaton, J. A., Henry, G. W., \& Fekel, F. C. 2003, in The Future of Small Telescopes in the New Millennium, Volume II: - The Telescopes We Use, ed. T. D. Oswalt (Dordrecht: Kluwer), 189

Fortney, J. J., Marley, M. S., \& Barnes, J. W. 2007, ApJ, 659, 1661

Gilliland, R. L., \& Baliunas, S. L. 1987, ApJ, 314, 766

Hall, J. C., Henry, G. W., Lockwood, G. W., Skiff, B. A., \& Saar, S. H. 2009, AJ, 138,312

Hall, J. C., Lockwood, G. W., \& Skiff, B. A. 2007, AJ, 133, 862

Henry, G. W. 1995a, in ASP Conf. Ser. 79, Robotic Telescopes: Current Capabilities, Present Developments, and Future Prospects for Automated Astronomy, ed. G. W. Henry \& J. A. Eaton (San Francisco, CA: ASP), 37

Henry, G. W. 1995b, in ASP Conf. Ser. 79, Robotic Telescopes: Current Capabilities, Present Developments, and Future Prospects for Automated Astronomy, ed. G. W. Henry, J. A. Eaton (San Francisco, CA: ASP), 44

Henry, G. W. 1999, PASP, 111, 845

Henry, G. W., Baliunas, S. L., Donahue, R. A., Fekel, F. C., \& Soon, W. 2000a, ApJ, 531, 415

Henry, G. W., Marcy, G. W., Butler, R. P., \& Vogt, S. S. 2000b, ApJ, 529, L41

Johnson, J. A., Winn, J. N., Cabrera, N. E., \& Carter, J. A. 2009, ApJ, 692, L100

Lissauer, J. J., \& Rivera, E. J. 2001, ApJ, 554, 1141

Mayor, M., et al. 2009, A\&A, 493, 639

Meschiari, S., Wolf, A. S., Rivera, E. J., Laughlin, G., Vogt, S. S., \& Butler, R. P. 2009, PASP, 121, 1016

Pace, G., \& Pasquini, L. 2004, A\&A, 426, 1021

Paulson, D. B., Saar, S. H., Cochran, W. D., \& Henry, G. W. 2004, AJ, 127, 1644

Perryman, M. A. C., et al. 1997, A\&A, 323, L49

Press, W. H., Teukolsky, S. A., Vetterling, W. T., \& Flannery, B. P. 1992, Numerical Recipes: The Art of Scientific Computing (2nd ed.; Cambridge, U.K.: Cambridge University Press)

Queloz, D., et al. 2001, A\&A, 379, 279

Queloz, D., et al. 2009, A\&A, 506, 303

Rivera, E. J., et al. 2005, ApJ, 634, 625

Sousa, S. G., et al. 2008, A\&A, 487, 373

Takeda, G., Ford, E. B., Sills, A., Rasio, F. A., Fischer, D. A., \& Valenti, J. A. 2007, ApJS, 168, 297

Valenti, J. A., \& Fischer, D. A. 2005, ApJS, 159, 141

Vogt, S. S., et al. 1994, Proc. SPIE, 2198, 362

Wright, J. T. 2005, PASP, 117, 675

Wright, J. T., Marcy, G. W., Butler, R. P., \& Vogt, S. S. 2004, ApJS, 152, 261 